Relations industrielles

Industrial Relations

\title{
Jean-Daniel Reynaud, Les règles du jeu. L'action collective et la régulation sociale
}

\section{Reynald Bourque}

Volume 46, numéro 2, 1991

URI : https://id.erudit.org/iderudit/050683ar

DOI : https://doi.org/10.7202/050683ar

Aller au sommaire du numéro

Éditeur(s)

Département des relations industrielles de l'Université Laval

ISSN

0034-379X (imprimé)

1703-8138 (numérique)

Découvrir la revue

Citer ce compte rendu

Bourque, R. (1991). Compte rendu de [Jean-Daniel Reynaud, Les règles du jeu. L'action collective et la régulation sociale]. Relations industrielles / Industrial Relations, 46(2), 480-482. https://doi.org/10.7202/050683ar

Tous droits réservés @ Département des relations industrielles de l'Université Laval, 1991
Ce document est protégé par la loi sur le droit d'auteur. L’utilisation des services d'Érudit (y compris la reproduction) est assujettie à sa politique d'utilisation que vous pouvez consulter en ligne.

https://apropos.erudit.org/fr/usagers/politique-dutilisation/ 
study of three jurisdictions in which comparable worth policies have been implemented: Minnesota, San José and Australia. Minnesota adopted a Pay Equity Act in 1982 under which comparable worth has been implemented for state government employees. Thus far, these employees have received three sets of comparble worth pay adjustments. In San José, as part of the two-year contract that settled a July 1981 municipal employees' strike, the city agreed to make comparable worth pay adjustments for certain predominantly female city jobs. Subsequent contracts included additional adjustments. Australia adopted comparable worth (equal pay for work of equal value) policy in 1972 covering employees in the public as well as private sectors.

Based on a detailed study of these three jurisdictions, the author reaches the following conclusions with regard to the wage and employment effects of comparable worth. The resuits for Minnesota and San José suggest that, other things being equal, the comparable worth adjustments adopted there raised pay of women relative to men by about 9.9 and 5.8 percentage points, respectively. While these wage gains are substantial, they did not eliminate all sex differences in pay. In Australia also, the initial wage gains for women were as large (about 10 percentage points). However, the effect wore off rather rapidly so that, in the long run, the wage gains have been negligible. The author finds the same experience to be true concerning the employment effects of comparable worth. Other things being equal, the comparable worth adjustments reduced employment in predominently female jobs relative to predominantly male jobs by about 3.5 and 6.7 percent in Minnesota and San José, respectively. However, these adverse effects were offset by the underlying trend in employment growth. Thus, the author points out that it is unlikely that anyone actually lost his or her job as a result of the comparable worth adjustment. Again in Australia, the immediate adverse employment effect of implementing equal value was of a similar magnitude but tended to be neglible in the long run.

Overall, the most interesting and useful contribution of the book consists of the detailed and methodologically sound case studies in Chapters 4 through 6 . The analysis in these chapters points to rather inconsequential effectiveness of comparable worth policies for reducing the male/female wage gap in long run. At the conceptual level, the book does devote three chapters to applying the conventional economic theory to comparable worth. But, in this regard, it breaks no new grounds.

Naresh C. Agarwal

McMaster University

Les règles du jeu. L'action collective et la régulation sociale, par Jean-Daniel ReYNAUD, Paris, Armand Colin, 1989, 306 p., ISBN 2-200-31256-3

Le dernier ouvrage de Jean-Daniel Reynaud est le couronnement d'une réflexion originale et vivifiante qui s'avère sans conteste l'une des contributions majeures des trois dernières décennies dans le domaine des relations industrielles. Cet auteur prolifique, adepte de la sociologie de Crozier, est connu surtout pour ses analyses sur la négociation collective et le syndicalisme. L'analyste brillant qu'est Reynaud se fait ici essayiste: à travers une lecture critique des classiques de la sociologie (Marx, Weber, Durhkeim, Parsons), il développe une analyse de la régulation sociale qui renvoie dos à dos les explications déterministes d'inspiration marxiste et structuro-fonctionnaliste.

L'objet de cet essai sociologique sur la régulation sociale n'est pas étranger à la problématique d'un Dunlop qui veut expliquer l'origine des règles générées par les systèmes de relations industrielles. Toutefois, à l'instar des tenants d'une approche pluraliste des relations 
industrielles (Flanders, Clegg), Reynaud reproche à Dunlop de s'intéresser davantage aux règles qu'à l'analyse des processus de régulation et de traiter le conflit industriel comme un accident de parcours plutôt que comme un ressort essentiel de la régulation sociale. Il s'oppose également à la conception de Durkheim voulant que la régulation sociale soit fondée sur les normes et les valeurs inscrites dans la conscience collective. Fidèle aux enseignements de Crozier, Reynaud considère que c'est dans l'action collective plutôt que dans la conscience collective qu'il faut chercher les fondements de la régulation sociale. Il reprend à son compte les thèses d'Olson à l'effet que l'action collective n'est pas la résultante de l'aggrégation des intérêts individuels; toute action collective comporte une part de contrainte, et la contrainte de la règle sur l'individu est liée à son appartenance à une collectivité. L'action collective et la régulation sociale entretiennent des rapports d'interdépendance: la règle est donc tout autant le produit que la condition de l'action collective.

Reynaud tire de ces analyses les postulats de base d'une approche stratégique de la régulation sociale: 1) les règles sociales sont contraignantes parce que constitutives d'une collectivité; 2) chaque groupe social produit sa propre régulation et la vie en société est la rencontre de plusieurs systèmes de règles; 3) une régulation commune à plusieurs groupes sociaux est le résultat de processus d'interaction et non le produit d'une conscience collective transcendante. Soulignant que le but premier de l'analyse sociologique est d'expliquer la dynamique et les combinaisons des différents systèmes de règles, il pose les jalons d'une analyse stratégique de la régulation sociale articulée sur trois propositions. La première veut que l'apprentissage des régulations soit un processus collectif continu mais non progressif, les changements de régulation procédant généralement d'une crise. Reynaud soutient également que tout groupe social cherche à autonomiser son champ d'action, ce qui entraîne des conflits de régulation dont l'enjeu est un territoire réel ou symbolique. La dernière proposition occupe une place centrale dans l'analyse de Reynaud; il convient selon lui de faire une distinction entre la régulation de contrôle externe et la régulation autonome produite par une collectivité. Cette distinction se fonde non seulement sur l'origine mais également sur les modalités de mise en oeuvre des régulations régissant une organisation. La régulation étatique est la principale forme de régulation de contrôle; la règle négociée est aussi une régulation de contrôle malgré son caractère de décision conjointe, la contrainte qu'elle institue relevant d'une autorité indépendante des groupes impliqués dans son élaboration.

L'auteur puise dans l'étude des relations industrielles de nombreux exemples de confrontation de systèmes de régulation. Ainsi, l'analyse stratégique met en évidence la coexistence au sein des organisations et dans les rapports de travail de règles formelles et informelles, du travail prescrit et du travail réel. Tout groupe social est porteur d'un projet spécifique de régulation qu'il cherche à imposer par son action; la régulation effective des systèmes de relations industrielles est donc une synthèse de différents projets de régulation tout comme le travail réel est un compromis entre les règles formelles et les règles informelles de l'organisation. La négociation collective constitue un système particulier de régulation puisque les acteurs collectifs directement concernés fixent conjointement les règles contractuelles liant les individus qu'ils ont mission de représenter; Reynaud assimile cette régulation conjointe à une délégation de l'autorité publique. Ce système de régulation conjointe a une caractéristique remarquable, le recours à la force étant généralement reconnu comme légitime dans certaines limites plus ou moins strictes fixées par le droit. La règle négociée est le fruit d'un affrontement institutionnalisé fondé sur des stratégies collectives, elle établit un compromis entre différents projets de régulation associés à des intérêts spécifiques.

L'idée directrice de l'ouvrage de Reynaud est que la société ne constitue pas un système social homogène fondé sur une régulation globale mais plutôt un ensemble hétérogène de systèmes sociaux instituant des régulations partielles et décentralisées. À l'appui de cette thèse, 
l'auteur se livre à une analyse critique des principales théories sociologiques postulant l'existence de systèmes sociaux générateurs de régulation globale. Le concept de statut social permet de classer les groupes sociaux selon une échelle de prestige et d'honneur mais Reynaud estime que la société actuelle est caractérisée par une pluralité des critères d'excellence difficilement compatible avec la thèse fonctionnaliste d'une hiérarchie sociale fondée sur des valeurs culturelles. L'analyse marxiste des rapports de production permet de définir les classes sociales mais l'action ouvrière s'exprime à travers une multitude de conflits catégoriels et localisés réflétant l'effritement de la conscience de classe. Reynaud considère par ailleurs que la vision néomarxiste de Touraine d'une lutte de classe débordant la sphère de la production pour englober tous les aspects de la vie sociale ne rend compte qu'imparfaitement de l'hétérogénéité et de l'autonomie des conflits sociaux. Le principal défaut des raisonnements fonctionalistes et marxistes serait selon Reynaud de supposer l'unité du système social, d'assimiler à une régulation globale l'équilibre instable résultant des interactions des différents systèmes sociaux.

Reynaud voit dans le déclin de l'État, et de l'action collective de masse, non pas la fin des idéologies proclamée par Aron et Bell mais plutôt un recul des régulations de contrôle au profit de systèmes de régulation plus modestes et décentralisés. Les valeurs, les croyances et l'action collective ne sont pas mortes, elles s'expriment sous des formes plus fragmentées que par le passé. L'auteur conclut son ouvrage en se demandant si une science des règles est possible. Les modèles des sciences physiques sont peu utiles en cette matière puisque la régulation sociale, fondée sur l'action collective et le compromis, ne se prête guère à la prévision. Dans les sciences humaines, la prévision tient davantage du conseil que de l'anticipation puisqu'elle influence les décisions des acteurs et peut orienter le cours de l'action; l'étude des règles relève pour l'essentiel du domaine d'une science des aides à la décision.

Cette présentation sommaire de l'ouvrage de Reynaud ne rend pas justice à la richesse des analyses de l'auteur. Elle se veut davantage une invitation à la lecture qu'un exposé rigoureux des thèses défendues par l'un des meilleurs représentants de l'école française des relations industrielles. Cet ouvrage ambitieux, voire même érudit, au cheminement parfois difficile pour le lecteur non averti, ne peut certes pas être considéré comme un manuel d'introduction aux relations industrielles. Il s'adresse plutôt à ceux et celles qui reconnaissent la contribution essentielle de la sociologie à cette entreprise multidisciplinaire qu'est l'étude des relations industrielles.

Reynald Bourque

Université du Québec à Hull

Vingt-cinq ans de pratique en relations industrielles au Québec, sous la direction de Rodrigue Blouin, Les Éditions Yvon Blais inc., Cowansville, Qué., 1990, 1164 p.,

ISBN 2-89073-715-2

Ce colossal ouvrage de plus de 1100 pages a été réalisé à l'occasion du vingt-cinquième anniversaire de la Corporation des conseillers en relations industrielles du Québec. Cet organisme professionnel a voulu ainsi trouver une façon originale et concrète de participer à l'histoire et à l'évolution des relations industrielles au Québec. $\mathrm{M}^{\mathrm{e}}$ Rodrigue Blouin, professeur au Département des relations industrielles et membre actif de la corporation fut chargé d'assumer la responsabilité de l'édition de l'ouvrage qui, l'on s'en doutera, est un collectif d'auteur-e-s et non l'oeuvre d'une seule personne.

Le volume comporte quatre parties. La première s'intitule: «La pratique en relations industrielles» et comprend neuf textes qui touchent les domaines suivants: les orientations en 